\title{
Magnetopause mapping to the ionosphere for northward IMF
}

\author{
I. I. Alexeev ${ }^{1}$, E. S. Belenkaya ${ }^{1}$, S. Yu. Bobrovnikov ${ }^{1}$, V. V. Kalegaev ${ }^{1}$, J. A. Cumnock ${ }^{2,3}$, and L. G. Blomberg ${ }^{2}$ \\ ${ }^{1}$ Skobeltsyn Institute of Nuclear Physics, Moscow State University, Moscow, Russia \\ ${ }^{2}$ Space and Plasma Physics, School of Electrical Engineering, Royal Institute of Technology, Stockholm, Sweden \\ ${ }^{3}$ Center for Space Sciences, University of Texas at Dallas, Richardson, TX, USA
}

Received: 2 March 2007 - Revised: 6 September 2007 - Accepted: 30 October 2007 - Published: 2 January 2008

\begin{abstract}
We study the topological structure of the magnetosphere for northward IMF. Using a magnetospheric magnetic field model we study the high-latitude response to prolonged periods of northward IMF. For forced solar wind conditions we investigate the location of the polar cap region, the polar cap potential drop, and the field-aligned acceleration potentials, depending on the solar wind pressure and IMF $B_{y}$ and $B_{x}$ changes. The open field line bundles, which connect the Earth's polar ionosphere with interplanetary space, are calculated. The locations of the magnetospheric plasma domains relative to the polar ionosphere are studied. The specific features of the open field line regions arising when IMF is northward are demonstrated. The coefficients of attenuation of the solar wind magnetic and electric fields which penetrate into the magnetosphere are determined.
\end{abstract}

Keywords. Magnetospheric physics (Auroral phenomena, Magnetospheric configuration and dynamics, Solar windmagnetosphere interactions)

\section{Introduction}

The polar cap can, as a first approximation, be considered a region void of precipitating particles inside the auroral oval. However, during northward interplanetary magnetic field (IMF), several different types of high-latitude emissions have been observed (Kullen et al., 2002), including transpolar aurora (TPA). The TPAs cross the noon-midnight meridian only when $B_{y}$ changes sign, resulting in a large $B_{z} /\left|B_{y}\right|$ ratio. The theta aurorae form in the Northern Hemisphere, from an expanded dawnside (duskside) emission region when $B_{y}$ changes from negative to positive (positive to negative) (Cumnock et al., 1997, and references therein).

Correspondence to: I. I. Alexeev

(iialexeev@mail.ru)
It is clear that energetic (up to several keV) particle precipitation is the cause of these emissions, although the source of them remains unknown. The precipitating particles may be accelerated by a magnetic field-aligned potential drop (Lyons 1980; Carlson and Cowley 2005), or may be a consequence of pitch-angle scattering of the magnetospheric plasma sheet particle populations due to stretched tail-like field lines at the current sheet. The visible and UV emissions can be driven by one or both mechanisms. Either way, the energy source of the auroral particle fluxes is the solar wind flow past the magnetosphere. The magnetospheric field line geometry is highly complex in the northward IMF case, as pointed out, for example, by Cowley (1973), Alexeev and Belenkaya (1983) (see also Siscoe et al., 2001), and Belenkaya (1998a, b).

Frank et al. $(1982,1986)$ proposed that theta aurora is located on closed field lines and is connected with the plasma sheet boundary layer or the distant plasma sheet. On the basis of particle flux data, Peterson and Shelley (1984) also supported the closed field lines idea. Reiff and Burch (1985) explained the high-latitude aurora under northward IMF, assuming open magnetic flux across the tail lobes.

Reconnection inside the magnetosphere for northward IMF was first described by Alexeev and Belenkaya (1983) and later called by Siscoe (1988) "the current penetration model". The reconnection x-line for northward IMF transforms into two magnetic field neutral points where 3-D reconnection occurs near the cusps (Alexeev and Belenkaya, 1983).

The portion of the IMF that penetrates into the magnetosphere is only a small part of the average magnetospheric magnetic field. However, this small field strongly controls the outer magnetosphere structure. For $B_{z}<0$, the reconnection region is located in the equatorial part of the dayside magnetopause, and in the magnetotail, while for $B_{z}>0$, two reconnection sites are located in the cusp regions (see, for example, Alexeev and Belenkaya, 1985). The consequences of the transformation from southward to northward

Published by Copernicus Publications on behalf of the European Geosciences Union. 


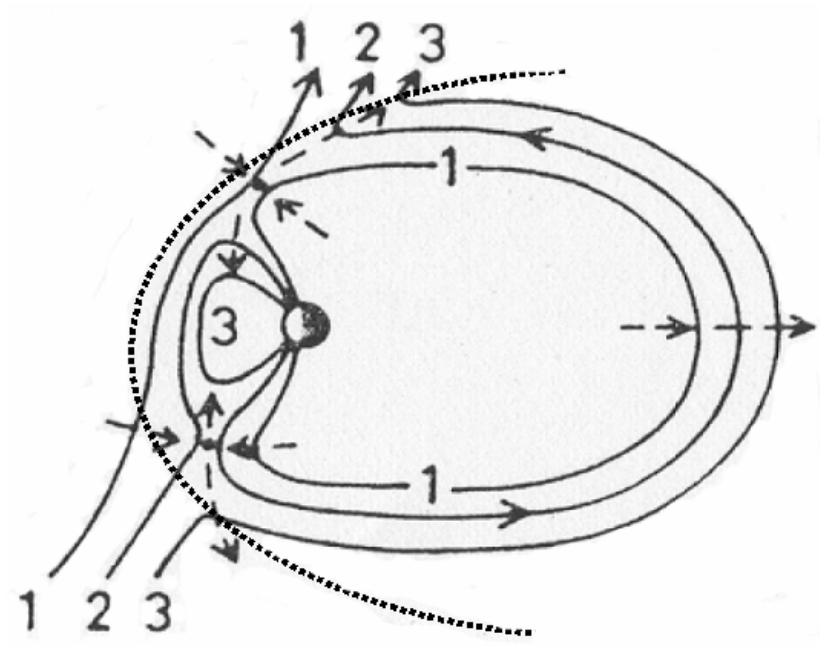

Fig. 1. We have followed Dungey's (1963) ideas which were later described by Cowley (1983), who represented the sequential merging in the Northern and Southern Hemisphere for the cases of IMF $B_{x}<0$. We have drawn on Fig. 1e from Cowley (1983) a dotted curve which indicates the magnetopause. Lobe reconnection first occurs in the Northern Hemisphere, forming an open field line that drapes over the dayside and one that connects to the Southern Hemisphere. Later, the overdraped field line merges with one of the previously opened field lines in the Southern Hemisphere, forming a closed dayside field line and a completely detached IMF field line (see also Belenkaya, 1998a, b).

IMF cases are not limited to moving the reconnection site from the equatorial dayside magnetopause to the cusp region. The overall topology of the IMF and magnetospheric field reconnection is different for northward and southward IMF directions (Alexeev, 1986; Belenkaya, 1998a, b).

The theme of this paper is internal reconnection for northward IMF. Our analysis is based on a long history of modeling of magnetospheric merging configurations, for example, see reviews by Siscoe (1988) and Crooker (1990). In this paper we refer to one of the simplest models classified by Siscoe (1988) as a current penetration model (Alexeev and Belenkaya, 1983) (see also Crooker, 1990, 1992; Crooker et al., 1998). In addition to superposing a dipole field and a uniform field (IMF) (Dungey, 1963; Cowley, 1973; Stern, 1973) this model associates the merging process with a magnetospheric boundary current through which the interplanetary magnetic field penetrates the magnetosphere. For northward IMF, at some point in the cusp region inside the magnetosphere, the penetrated IMF and the magnetospheric field cancel each other. At the neutral point of the magnetic field three-dimensional reconnection occurs. A short description of this model is given in the next section.

We follow the line of Dungey (1963) and Cowley (1983). In our approach, similar to the northward IMF reconnection scenario shown in Fig. 1 from Cowley (1983), two neutral points exist near the northern and southern cusps. The open field lines appear at one neutral point, as a result of reconnection of the IMF lines penetrated into the magnetosphere and closed field lines. Later, the open field lines of both polar caps reconnect, forming closed and IMF field lines. The reconnection for different IMF orientations is considered by us in detail in a set of papers for the Terrestrial, Jovian and Kronian magnetospheres (e.g. Alexeev and Belenkaya, 1985, 1989; Belenkaya, 1993, 1998a, b, 2004; Belenkaya et al., 2006; Belenkaya et al., 2006a, b).

Figure 1 presents Cowley's (1983) representation of sequential merging in the Northern and Southern Hemispheres (see Fig. 1e in Cowley, 1983). Lobe reconnection first occurs in the Northern Hemisphere, forming an open field line that drapes over the dayside and one that connects to the Southern Hemisphere. Later, the overdraped field line merges with one of the previously opened field lines in the Southern Hemisphere, forming a closed dayside field line and a completely detached field line. Similar sequential merging can also occur in the case of open field lines in the tail, though this is not illustrated in the Fig. 1.

We assume that, with the exception of a relatively small diffusion region near the neutral point, the magnetic field lines could be considered as equipotential. In the diffusion region, a field-aligned potential drop appears. Here we concentrate on quantitative calculations of the magnetic and electric fields near the high-latitude reconnection site for northward IMF. Based on the dynamic magnetospheric model, quantitatively taking into account the IMF penetration into the magnetosphere, we will map the solar wind electric potential along the magnetic field lines to ionospheric altitudes.

\section{Model description}

The current penetration model of the IMF and magnetospheric magnetic field merging was proposed by Alexeev and Belenkaya (1983) and was later developed by Crooker et al. (1990) (see also Siscoe, 1988). As a consequence of the high (but not infinite) solar wind plasma conductivity, $\sigma$, the normal component of the magnetopause magnetic field is nonzero, and proportional to $R_{m}^{-1 / 4}$ (Alexeev, 1986). The magnetic Reynolds number, $R_{m}=\mu_{0} \sigma V R_{1}$, is determined by the magnetopause subsolar distance, $R_{1}$, by the solar wind velocity $V$, and by the effective conductivity $\sigma$ of the solar wind plasma. The solution inside the magnetosphere, found by Alexeev (1986), is a spatially uniform magnetic field penetrated into the magnetosphere, given by $B_{n} \sim R_{m}^{-1 / 4}$.

Dorelli et al. (2004) also demonstrated that the annihilation rate scales, for instance, like $R_{m}^{-1 / 4}$ for magnetic reconnection at the terrestrial subsolar magnetopause. Using global MHD simulations, Dorelli et al. (2004) supported the Alexeev (1986) result $\left(B_{n} \sim R_{m}^{-1 / 4}\right)$, which was obtained analytically, both for a simpler kinetic approach and for the parabolic magnetopause shape. Dorelli et al. (2004) 


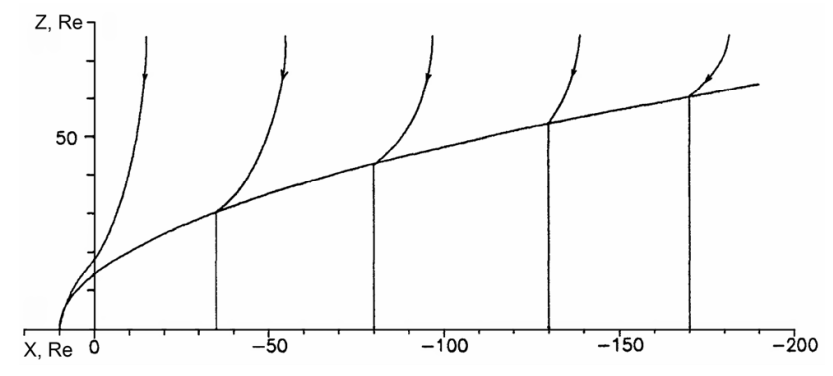

Fig. 2. Plasma flow lines and magnetic field lines in the $y=0$ plane. The $\mathrm{x}$-axis is the Earth-Sun line, the $\mathrm{z}$-axis is parallel to the IMF vector upstream of the bow shock. This coordinate system can be obtained from the solar-magnetospheric coordinates by a rotation around the $\mathrm{x}$-axis. In solar-magnetospheric coordinates, the IMF vector is $\left\{B_{x}, B_{y}, B_{z}\right\}$. In IMF-magnetospheric coordinates the IMF vector is $\left\{B_{x}, 0, B t\right\}$, where $B_{t}=\sqrt{B_{y}^{2}+B_{z}^{2}}$.

demonstrated that if the plasma resistivity is spatially uniform, magnetic reconnection occurs in the thin current sheet at a rate which is consistent with that predicted by the analytical, incompressible flow solutions (though there are small corrections in simulations due to the plasma compressibility).

Let us demonstrate the IMF draping of the solar wind flow past the magnetosphere for high conductivity of the solar wind plasma. In Fig. 2 (from Alexeev and Kalegaev, 1995) a cross section of the magnetosphere parallel to the IMF (the IMF vector lies in the XZ plane of Fig. 2) is shown. As the magnetospheric magnetic field is precluded from penetrating outside by the magnetopause currents, the magnetic field lines in the magnetosheath do not depend on the mutual orientation of the IMF and geomagnetic dipole. Outside the magnetopause, the draping magnetic field ( $\left.B_{\mathrm{IMF}}\right)$ can be calculated as a sum of two terms: magnetic field penetrated into the magnetosphere, $b$, and the part of IMF which is screened by the currents at the magnetopause current layer, $B_{\mathrm{scr}}$ :

$B_{\mathrm{IMF}}=b+B_{\text {scr }}$.

Both parts of the IMF ( $b$ and $B_{\text {scr }}$ ) have been obtained by Alexeev et al. (1998). Inside the magnetosphere the solution of the IMF penetration problem is a uniform magnetic field:

$b=k_{\|} B_{0 \|}+k_{\perp} B_{0 \perp}$,

where $B_{0 \| \mid}$ and $B_{0 \perp}$ are the IMF components tangential and normal to the undisturbed solar wind velocity, $V$, and $k_{\|}$and $k_{\perp}$ are the screening coefficients for the corresponding IMF components

$\left(k_{\perp}=0.9 \chi^{1 / 2} R_{m}^{-1 / 4}\right.$, and $k_{\|}=2 \chi\left(\pi R_{m}\right)^{-1 / 2}$, respectively). These coefficients depend on the magnetic Reynolds number $R_{m}$, and on the parameter $\chi=\rho_{2} / \rho_{1}$, which determines the plasma compression at the bow shock (Alexeev et al., 2003); here $\rho_{1}$ is the solar wind plasma density, and $\rho_{2}$ is the magnetosheath plasma density downstream of the bow shock. The

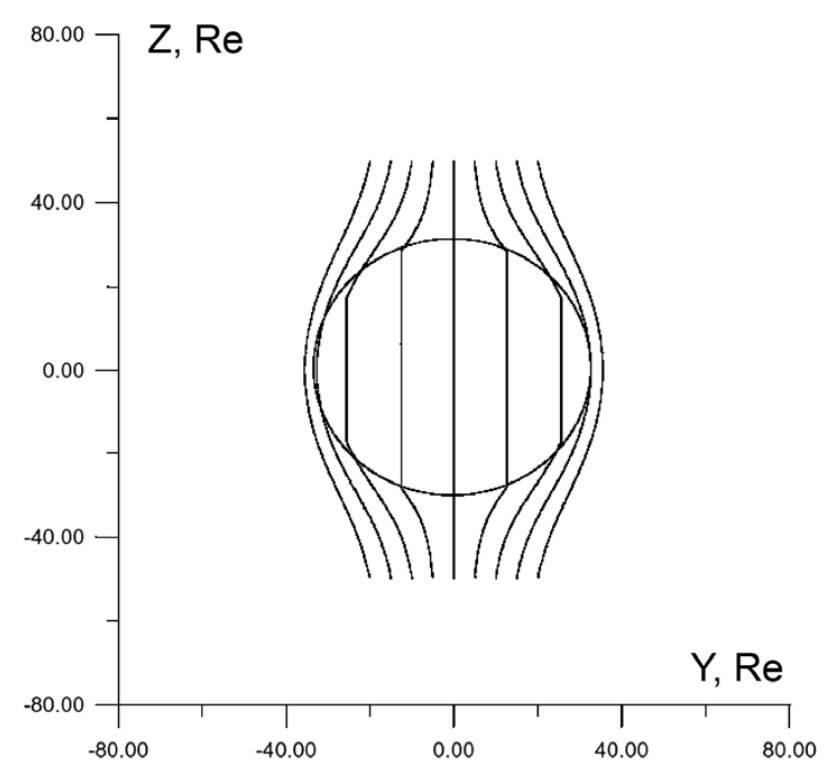

Fig. 3. A projection of the IMF lines on the plane $x=R_{1} / 2$ (where the paraboloid focus is located) is shown. We see the draping of the magnetic field lines. As a result, the IMF penetrated into the magnetosphere, $b=\kappa_{B} B$, is only a small part of the IMF upstream of the bow shock. The factor $\kappa_{B}$ is about 0.1 (Alexeev, 1986), and it describes the weakening of the IMF inside the magnetosphere.

IMF distortions in the plane perpendicular to the Sun-Earth line are shown in Fig. 3. In this figure the projections of the magnetic field lines on the $\mathrm{YZ}$ plane are drawn. We see that as a result of these distortions, only a small part of the electric field potential drop at the magnetospheric diameter can be applied to the polar cap. The effective cross-sectional area of the magnetosphere perpendicular to the Sun-Earth line is smaller than the transverse dimension of the geometric magnetosphere by a factor $\kappa_{B}$, equal to the ratio of the penetrated portion of the IMF to the upstream solar wind magnetic field, $\kappa_{B}=k_{\perp}$, is the dimensionless factor which describes a depression of the interplanetary magnetic field ( $B_{z}$ and $B_{y}$ components), penetrating the magnetosphere (see Eq. 2). The weakening of the solar wind velocity in the boundary layer inside the magnetosphere, near the magnetopause, gives an additional decrease of the penetrated solar wind electric field in $\kappa_{v}$ times. This leads to a field-aligned potential drop in the boundary layer region.

The total picture of the IMF penetrating into the magnetosphere for southward IMF is shown in Fig. 4 from Alexeev et al. (1998). The magnetosphere - solar wind interaction is strongly controlled by the IMF direction, and it is significantly different for northward and southward IMF. However, the disturbances of the IMF and plasma flow in the magnetosheath are similar for both IMF directions in the zero approximation. The undisturbed solar wind region is shown upstream of the bow shock (left side of Fig. 4). Here 


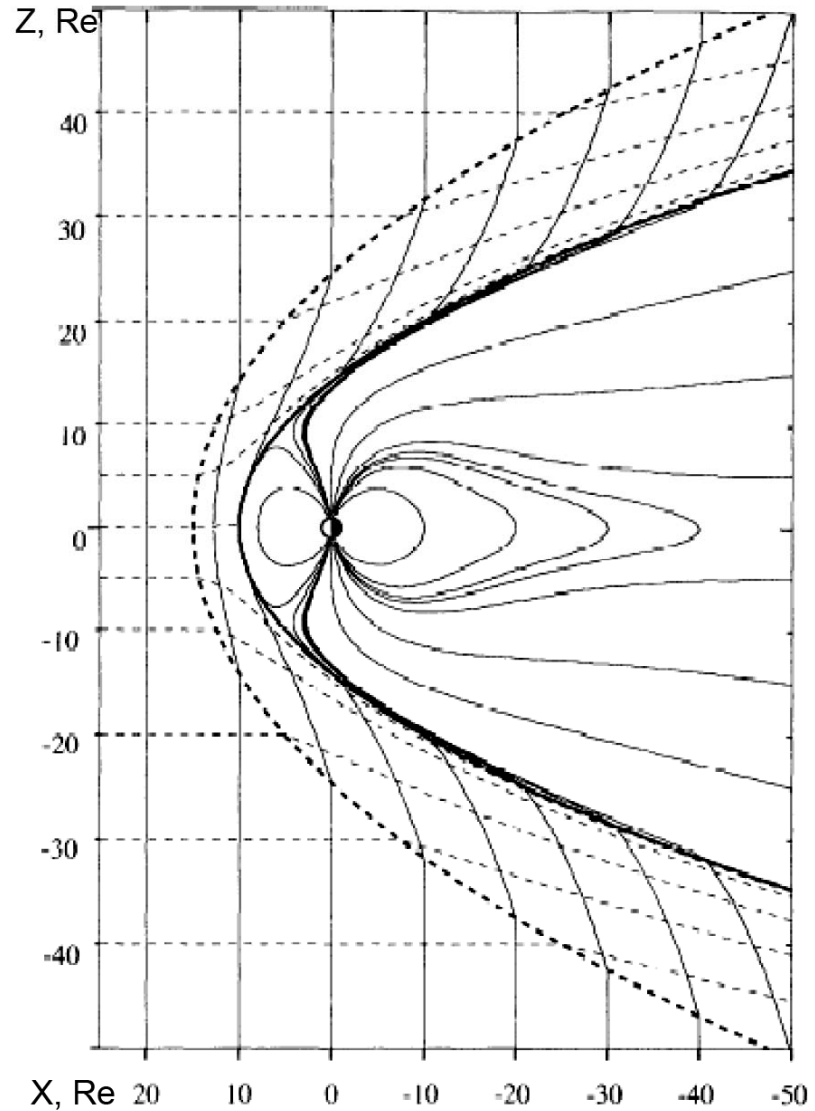

Fig. 4. The interplanetary and magnetospheric magnetic field lines in the noon-midnight plane are shown (thin solid curves), as well as the magnetopause (thick solid curve). Upstream of the bow shock and in the magnetosheath we also see the streamlines of the solar wind flowing past the magnetosphere (thin dashed curves).

the velocity and magnetic field are uniform. After the bow shock crossing, the IMF draping by the bow shock and magnetopause currents is seen. Inside the magnetosphere, we see the shielded field of the internal sources summed with the penetrated IMF $b$.

In the paraboloid model we represent the magnetopause as a paraboloid of revolution, which is axially symmetric relative to the Sun - Earth line. We will calculate the field lines which cross both the magnetopause and the ionosphere (the open field lines). These field lines can cover only a part of the polar cap; the other part of the black area inside the auroral oval can be crossed by closed field lines (connecting in both hemispheres) which extend into the distant tail.

The model used here is based on the results of Alexeev and Belenkaya (1983), Alexeev (1986), and Belenkaya (1998a, b). For northward IMF, detached IMF lines exist inside the magnetosphere; they intersect the magnetopause current sheet two times and do not reach the Earth's surface (see Fig. 2 in Blomberg et al., 2005). Correspondingly, between the magnetopause current sheet and the open field line bundle connected with the Earth a bundle of interplanetary magnetic field lines penetrated into the magnetosphere appears. As we speculate here, this is one of the main differences between the southward and northward IMF cases. The reason for this effect is the following: For a closed magnetosphere (when IMF is equal to zero), two neutral points lie on the magnetopause (cusps). For northward IMF, these magnetic nulls cannot stay at the magnetopause. Inside the magnetopause, near the northern cusp, a magnetic field with a north-south component directed to the Earth exists. When the penetrated IMF $b_{z}=\kappa_{B} B_{z}>0$ is added to the magnetospheric field the northern null moves inside the magnetopause. The coefficient of IMF penetration into the magnetosphere is $\kappa_{B} \sim 0.1$ (Alexeev, 1986). Due to a partial IMF penetration inside the magnetosphere, magnetic field neutral points should be shifted inside the magnetosphere to satisfy a condition $B=0$. Here we take into account the screening effects of the solar wind plasma. Near the magnetic field nulls can be essential in penetrated into the magnetosphere or trapped near cusp plasma. If we study the plasma term by test particle analysis we can show that ion currents only increase northward if $b_{z}$.

Contrary to the northward IMF case, for southward IMF, at the equatorial magnetopause the magnetic field vectors at the magnetosheath and magnetospheric sides of the magnetopause have opposite directions and a neutral line (connected noon and midnight reconnection sites) is placed inside the magnetopause and tail current sheet.

We follow Dungey's (1963) ideas which were later developed by Cowley (1983), who have represented the sequential merging in the Northern and Southern Hemispheres for the case of IMF $B_{x}<0$ which will be studied here. In this case, the high-latitude reconnection first occurs in the Northern Hemisphere, forming the open field lines connected to the Northern and Southern polar caps. Later, these field lines merge in the Southern Hemisphere, forming closed field lines and completely detached IMF field lines (see also Belenkaya, 1998a, b).

In Alexeev and Belenkaya (1983) it was shown for the spherical and paraboloid magnetospheric model that for northward IMF two magnetic nulls are found inside the magnetosphere (later this concept was developed by Alexeev, 1986 and Belenkaya, 1998a, b). This is an advantage of our model compared to MHD models, for which it is difficult to determine the magnetopause position. For example, Siscoe et al. (2001) have identified the magnetopause with the open/closed field line boundary.

The layer of IMF lines penetrated into the magnetosphere for northward IMF moves the lobe reconnection site from the magnetopause inside the magnetosphere (into the boundary layer usually observed at the magnetospheric side of the magnetopause). As a result, the plasma flow velocity is $\kappa_{v} V$, where $V$ is the solar wind velocity and the coefficient $\kappa_{v}<1$ describes a reduction in the plasma velocity in the boundary layer at the lobe reconnection site. The IMF penetrated into the magnetosphere, $b$, is only a small part of the IMF 
strength upstream of the bow shock $\left(b=\kappa_{B} B\right)$. The product of both coefficients $\kappa_{v} \kappa_{B}=\kappa_{E}$ gives the solar wind electric field weakening inside the magnetosphere (see Eq. 3).

By mapping the electric potential from the magnetopause to the polar cap along open field lines, we obtain the electric potential distribution in the magnetosphere (Alexeev, 1986; Alexeev et al., 1989; Toffoletto and Hill, 1989). The approach $\Delta \Phi_{\|}=0$ is valid everywhere for the open field lines bundle, excluding only those that intersect the diffusion region near the neutral points. The potential drop across the polar cap is determined in terms of the solar wind parameters as follows:

$\Delta \Phi_{p c}=\kappa_{B} \kappa_{v} V B_{t} L_{y}=\kappa_{E} \Delta \Phi_{m}$.

Here $B_{t}=\sqrt{B_{y}^{2}+B_{z}^{2}}$ is the IMF component, perpendicular to the solar wind velocity, $L_{y}$ is the transverse dimension of the region of open field lines on the magnetopause, $\Delta \Phi_{m}=V B_{t} L_{y}$ is the potential drop across the magnetosphere determined by the undisturbed solar wind flow upstream of the bow shock. The numerical value of $\kappa_{B}$ is determined by the solar wind velocity and density, and the empirical value of the solar wind plasma conductivity $\sigma$. As deduced by Alexeev et al. (2000):

$\kappa_{B}=0.9\left(\mu_{0} \sigma\right)^{-1 / 4}\left(V R_{1}\right)^{-1 / 4}$,

where $\mu_{0}$ is the vacuum permeability, and $R_{1}$ is the geocentric distance to the subsolar point. Such a decrease in the penetrated interplanetary field is a consequence of the high solar wind plasma conductivity, $\sigma$. If the magnitude of conductivity $\sigma$ is $2 \times 10^{-4}(\mathrm{Ohm} \mathrm{m})^{-1}$, then $\kappa_{B}=a_{1}\left(V R_{1}\right)^{-1 / 4}$, where the factor $a_{1}$ is equal to $0.22 \times 10^{3} \mathrm{~m}^{1 / 2} \mathrm{~s}^{-1 / 4}$ (Alexeev et al., 2000). Finally, Alexeev et al. (2000) gave for southward $B_{z}$ the dependence of $\Delta \Phi_{p c}$ on $B_{z}$ and on the solar wind plasma parameters, i.e. density $(n)$ and velocity $(V)$ :

$\Delta \Phi_{p c}(\mathrm{kV})=1.25 B_{z}(n T)\left(n\left(\mathrm{~cm}^{-3}\right)\right)^{-1 / 8}(V(\mathrm{~km} / \mathrm{s}))^{1 / 2}$.

For the typical values of $n=5 \mathrm{~cm}^{-3}, V=400 \mathrm{~km} / \mathrm{s}$, and $B_{z}=-5 \mathrm{nT}$, Eq. (5) gives $\Delta \Phi_{p c}=102 \mathrm{kV}$, here $L_{y}$ equals $8 R_{1}$ as was demonstrated by our model calculations, and $\kappa_{B}=0.1$. For similar solar wind parameters, but IMF in the dawn-dusk direction, Siscoe et al. (2001) gave $49 \mathrm{kV}$ as an estimation of the reconnection potential drop in the MHD simulations. Siscoe et al. (2001) have used a global MHD simulation to compute the distribution of $E_{\|}$on the face of the magnetopause, as represented by the last closed field line surface.

Using Eq. (3) with a factor $\kappa_{v}=0.5$, which takes into account the decreasing solar wind plasma flow velocity at the reconnection site for northward $B_{z}$, we finally obtained:

$\Delta \Phi_{p c}(\mathrm{kV})=0.625 B_{z}(n T)\left(n\left(\mathrm{~cm}^{-3}\right)\right)^{-1 / 8}(V(\mathrm{~km} / \mathrm{s}))^{1 / 2} .(6)$

For the solar wind parameters used by Siscoe et al. (2001) for simulations: density $5 \mathrm{~cm}^{-3}$, speed $350 \mathrm{~km} / \mathrm{s}$, and IMF strength $5 \mathrm{nT}\left(B_{x}=B_{z}=0\right)$, Eq. (6) gives $\Delta \Phi_{p c}=45 \mathrm{kV}$ comparable to the Siscoe et al. (2001) MHD simulation results which found the global reconnection rate to be $\sim 49 \mathrm{kV}$.

In the next section we discuss the model calculation results for the two cases with large $B_{y}$, which have also been studied by Cumnock and Blomberg (2004), Blomberg et al. (2005) and Cumnock et al. (2006). In this paper the ratio of the ionospheric polar cap potential drop to the potential drop across the magnetosphere determined by the undisturbed solar wind flow upstream of the bow shock is assumed to be $\kappa_{E}=\kappa_{B} \bullet \kappa_{v}=0.05$ (see Eq. 3).

\section{Model calculation results}

During the course of the events that are described below (on 8 November 1998), the IMF strength and solar wind dynamic pressure were both above their respective average values (up to $27 \mathrm{nT}$ and $20 \mathrm{nPa}$, respectively). The model calculations take into account not only the IMF influence on the structure of the magnetosphere, but also the dynamics of the subsolar distance and of the polar cap and auroral oval boundaries (see Alexeev et al., 2003). The model used here is parameterized by solar wind data from the ACE spacecraft and by the geomagnetic indices $D s t$ and $A L$ (obtained from the World Data Center in Kyoto). The Dst is used as an indicator of the ring current strength, and $A L$ is used for estimation of the disturbance level of the tail lobe magnetic flux (see Alexeev et al., 2003).

In Fig. 5 we show magnetic field data from the ACE (Advanced Composition Explorer) spacecraft that orbits the L1 Lagrange point approximately $220 R_{E}$ sunward of the Earth. IMF GSM components are plotted for 8 November 1998 (981108), as a function of corrected universal time (UT plus the estimated propagation time from the satellite to GSM $X=0$ ); in these events the time lag is about $45 \mathrm{~min}$.

IMF $B_{z}$ was positive for at least $3 \mathrm{~h}$ prior to and following a change in $B_{y}$. IMF $B_{x}$ was negative through the time period of interest. $B_{y}$ was small during $1-1.5 \mathrm{~h}$ while changing sign. ACE plasma measurements (not shown) indicate that during the time of the IMF $B_{y}$ change, the solar wind ion density was about $20 \mathrm{~cm}^{-3}$ and the solar wind velocity was approximately $550 \mathrm{~km} / \mathrm{s}$.

For the model calculations of the open field line bundles we have chosen two moments of time: case 1 at 13:18 UT on 8 November 1998, when the IMF components were $B_{x}=-10 \mathrm{nT}, B_{y}=10 \mathrm{nT}$, and $B z=23 \mathrm{nT}$ (the declination angle between the projection of the IMF onto equatorial projection and Earth-Sun line is equal to $135^{\circ}$ ); and case 2 at 15:00 UT on the same day when the IMF components were $B_{x}=-8 \mathrm{nT}$, $B_{y}=-14 \mathrm{nT}, B_{z}=22 \mathrm{n} T$. For case 1 , IMF was directed along the average direction of IMF (along the Archimedes spiral, $\left.B_{x}<0, B_{y}>0\right)$. Case 2 was chosen for the moment when $B_{y}$ had a negative sign and the projection of the IMF on the 


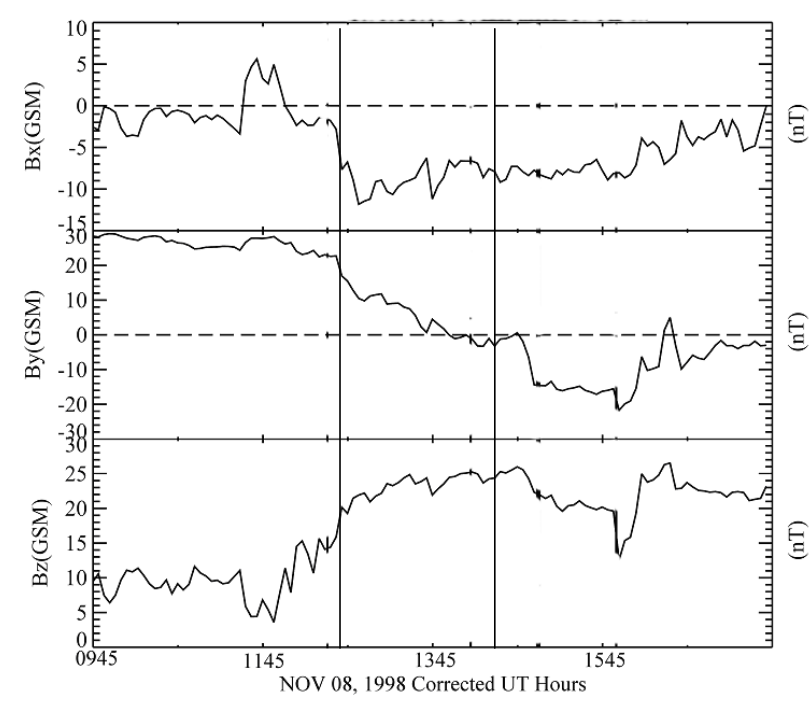

Fig. 5. Magnetic field data are shown from the ACE satellite which orbits the L1 Lagrangian point about $220 R_{E}$ sunward of the Earth. IMF GSM components are plotted for 8 November 1998, as a function of corrected universal time (UT plus the estimated propagation time from the satellite to GSM X=0); about $45 \mathrm{~min}$. The two cases studied correspond to the times marked by thin vertical lines.

equatorial plane $(\mathrm{Z}=0)$ was perpendicular to its average direction $\left(B_{x}<0, B_{y}<0\right)$.

In general, reversal of the sign of $B_{y}$ corresponds to a mirroring about the noon-midnight plane. The smaller difference between the northern and southern polar cap potential drops for case 2, compared to that for case 1, can be explained by the smaller ratio of IMF $B_{x}$ to $B_{y}$ for case 2 and the corresponding diminished role of the preferred hemisphere which is directly connected to the solar wind by open field lines. For this reason, the polar cap is more easily and more quickly populated by solar energetic particles during the solar cosmic ray enhancements.

For mapping of the solar wind electric field to the polar ionosphere using field line tracing, the $D s t$ and $A L$ geomagnetic indices are needed. The studied events correspond to quiet or slightly disturbed conditions. Dst was about $-50 \mathrm{nT}$ and $A L$ was $-400 \mathrm{nT}$ and $-69 \mathrm{nT}$, respectively, for the two instants modeled. The model polar cap magnetic flux changed from 740 to $430 \mathrm{MWb}$ between the cases. For case 1, 13:18 UT on 8 November 1998, the solar wind plasma parameters used in the model calculations are: density $21 \mathrm{~cm}^{-3}$, and velocity $572 \mathrm{~km} / \mathrm{s}$. For case $2,15: 00$ UT on 8 November 1998, the solar wind plasma parameters are: density $25 \mathrm{~cm}^{-3}$, and velocity $537 \mathrm{~km} / \mathrm{s}$, and correspond to the subsolar distance $R_{1}=7.9 R_{E}$.

The general view of the structure of the open field line bundles is given in Fig. 6 (upper panel) for case 1. Figure 6 (upper panel) shows the open magnetic field lines projection on the YZ plane of the solar-magnetospheric coordinate system.
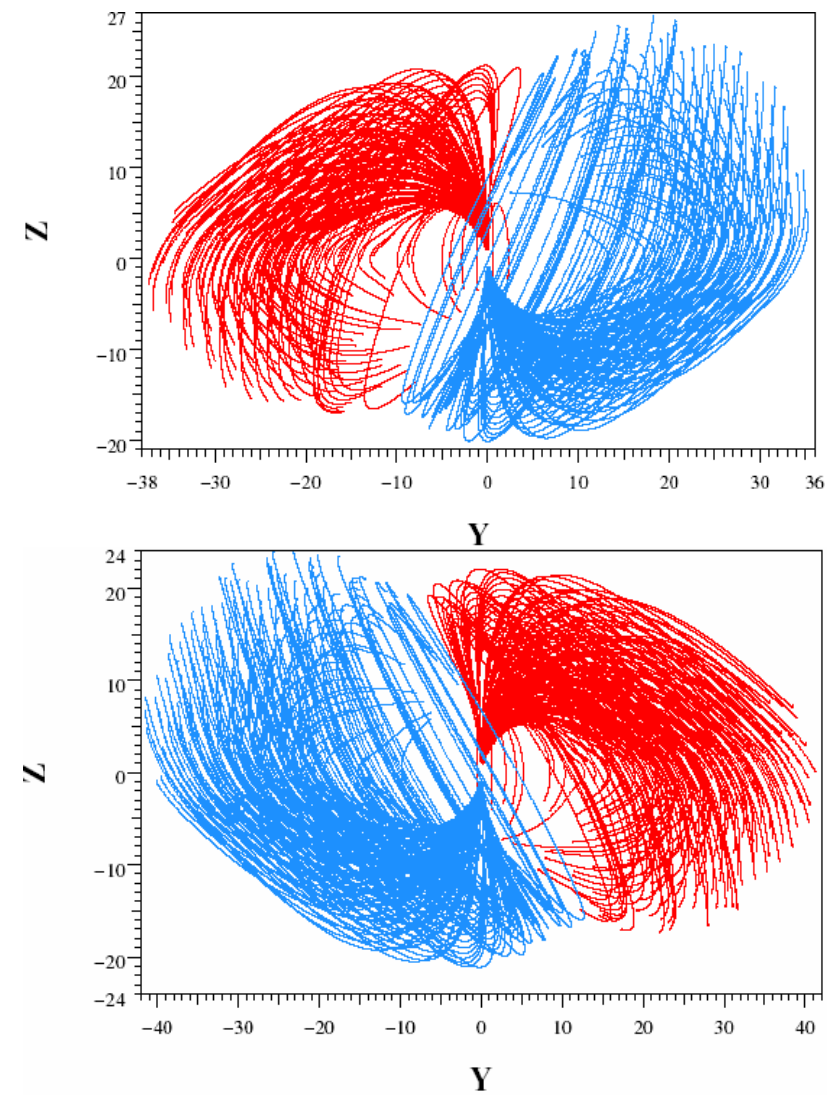

Fig. 6. View from the Sun to the open field line bundles. Upper panel: Case 1. The IMF components are: $B_{x}=-10 \mathrm{nT}, B_{y}=10 \mathrm{nT}$, and $B_{z}=23 \mathrm{nT}$, the same as for Fig. 4. The parts of the open field lines which are placed inside the magnetosphere are shown. Red field lines map from the magnetopause to the northern polar ionosphere, and blue field lines map from the southern polar ionosphere to the magnetopause. Outside the magnetopause (not shown) the red lines go from the Sun to the Earth and the blue lines go antisunward. Bottom panel: Case 2. Open field line bundles. All calculations are made for case 2: 15:00 UT on 8 November 1998, when the IMF components, according to the shifted ACE data, were $B_{x}=-8.2 \mathrm{nT}$, $B_{y}=-14 \mathrm{nT}, B_{z}=22 \mathrm{nT}$. The solar wind plasma parameters which were used in the model calculations are: the density $15 \mathrm{~cm}^{-3}$, and velocity $519 \mathrm{~km} / \mathrm{s}$ for this case.

Northern open field lines are shown by red curves and southern open field lines are shown by blue curves. From the dawn (dusk) south (north) magnetopause open field lines go to the northern (southern) polar cap. The feet of the field lines are mapped into the polar ionosphere with equal steps along the $x$ and $y$-axes. In Fig. 6 all magnetic field lines are shown that pass from the ionosphere toward the magnetopause. The two closest red and blue lines illustrate the form of the separator line, which connects both neutral points. All other field lines (not shown in Fig. 6) are closed. These closed field lines go from one hemisphere to the other. 
In Fig. 7 (upper panel) a view of the magnetopause surface from the Sun is shown for case 1.

The bottom panel of Fig. 7 shows the same view of the magnetopause, as for case 2. This figure demonstrates the spatial regions where the open field line bundles intersect the magnetopause. The projection of the IMF on the viewing plane is perpendicular to the Sun-Earth line. The IMF is directed from the bottom left side to the upper right side of the figure ( $B_{z}>0$ and $\left.B_{y}>0\right)$. The inclined lines in Fig. 7 show constant solar wind electric potentials at the magnetopause. As the potential drops on the northern (red) and southern (blue) open field line, bundles are multiplied by a factor $\kappa_{B}$ (see Fig. 2); they are smaller than those on the same scales upstream of the bow shock. Red and blue crosses show the points of intersection of the open field lines with the model magnetopause. These points were calculated by tracing the field lines from the polar ionosphere to the magnetopause.

Contrary to the southward IMF case, in Fig. 7, the incoming (red) open field line "window" at the magnetopause is separated from the out-going (blue) open field line "window". Calculation results show that the reconnection potential drops in the southern and northern hemispheres may be different, because the sizes of the red and blue windows in the direction perpendicular to the IMF are different. Only real model field lines which have been calculated numerically are shown in Fig. 6. There are no separator lines through these field lines. The separator lines go from one neutral point to the other neutral point. For each step, any infinitely small displacement from the correct separator line will lead to the passage to the ordinary field line which does not cross neutral points. The shape of the separator can be understood if one compares the red and blue field lines in Fig. 6. The separator field lines are not connected with the ionosphere and are placed at the mutual boundary of the northern and southern open field line bundles. In other words, a separator belongs to both field line bundles.

Before reconnecting in the northern or southern lobe neutral points, the IMF lines come in contact with the magnetopause (current sheet which bounds the magnetospheric magnetic field), then move inside the magnetosphere. At the moment of reconnection, IMF lines cross the bottom (upper) part of the blue (red) curve. This part of the blue (red) curve corresponds to the boundary of the southern (northern) open field line bundle. These regions of the open field lines bundles are mostly located sunward and are near the penetrated IMF. At the moment of reconnection, the IMF lines meet closed magnetospheric field lines and after reconnection form open field lines. After the movement across the open field lines window along the equipotential lines to the upper (bottom) part of the boundary, the open field lines meet the open field lines from the other hemisphere and form the IMF and closed field lines. The current penetration mechanism is connected to the magnetic field diffusion. Internal reconnection occurs between topologically "closed" geomagnetic field lines and that fraction of the IMF which has
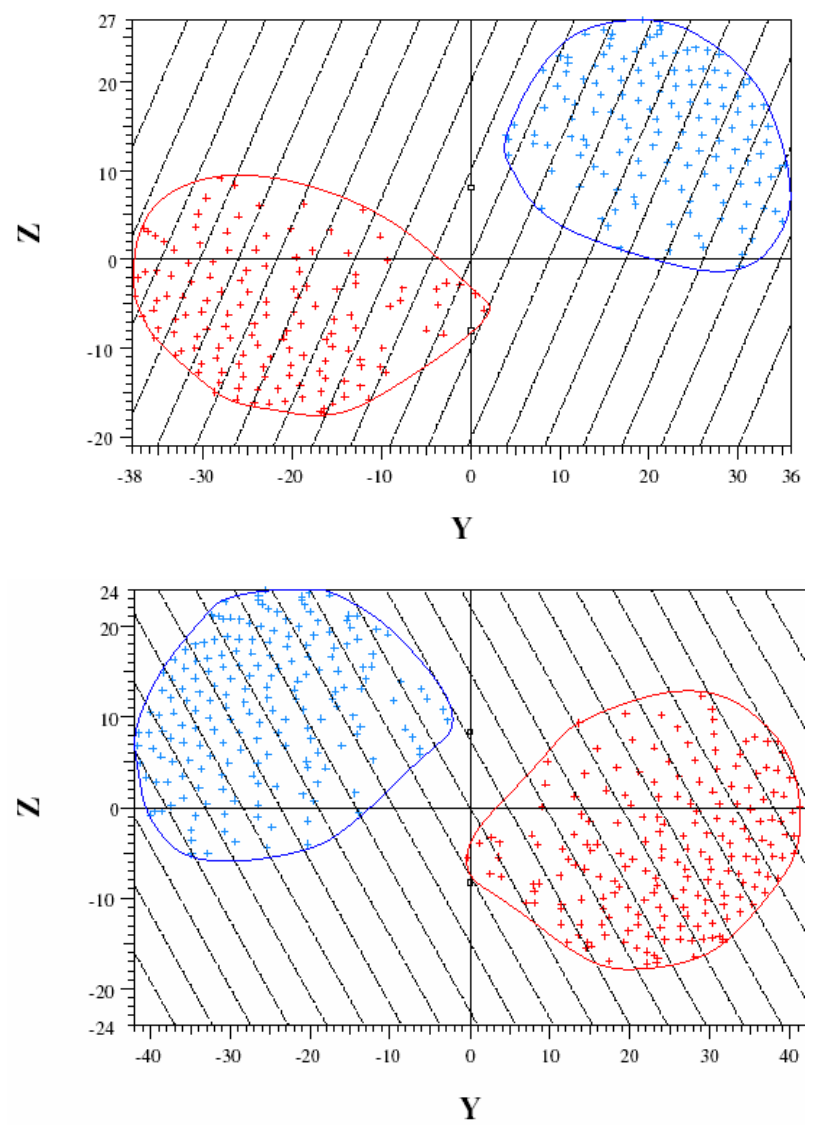

Fig. 7. View from the Sun towards the Earth's magnetosphere. The electric equipotentials are shown by the dashed lines on the magnetopause. The intervals between the two inclined lines are equal to $4 R_{E}$ and the potential drop between these two lines is equal to $16 \mathrm{kV}$. The region bounded by the red (blue) curve shows the intersection of the southern/dawn (northern/dusk) magnetopause with the open field line bundle of the northern (southern) polar cap. The parts of the equipotential lines on the magnetopause which are placed inside these regions project to convection cells in the ionosphere. Upper panel: Case 1. All calculations were made for the case 1: 13:18 UT on 8 November 1998, when, according to the shifted ACE data, the IMF components were $B_{x}=-10 \mathrm{nT}$, $B_{y}=10 \mathrm{nT}$, and $B_{z}=23 \mathrm{nT}$. The solar wind plasma parameters used in the model calculations are: density $21 \mathrm{~cm}^{-3}$ and velocity $572 \mathrm{~km} / \mathrm{s}$. Bottom panel: Case 2. All calculations are made for case 2: 15:00 UT on 8 November 1998, when the IMF components, according to the shifted ACE data, were $B_{x}=-8.2 \mathrm{nT}, B_{y}=-14 \mathrm{nT}$, $B_{z}=22 \mathrm{nT}$. The solar wind plasma parameters which were used in the model calculations are: the density $15 \mathrm{~cm}^{-3}$, and velocity $519 \mathrm{~km} / \mathrm{s}$ for this case.

diffused through the magnetopause current sheet. This is different from a lobe reconnection scenario between the already open lobe magnetic field and the IMF. For experimental data regarding the reconnection process for northward IMF for both of these scenarios, see Belenkaya, 1993. The strong control of the magnetospheric shape and dynamics by IMF $B_{z}$ can serve as evidence of IMF penetration into magnetosphere. 


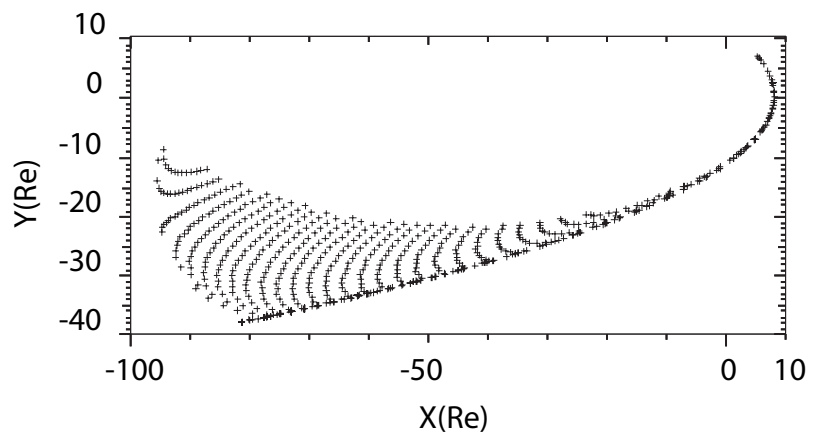

Fig. 8. Points of intersection of the northern polar cap open field lines with the equatorial plane, shown by crosses.
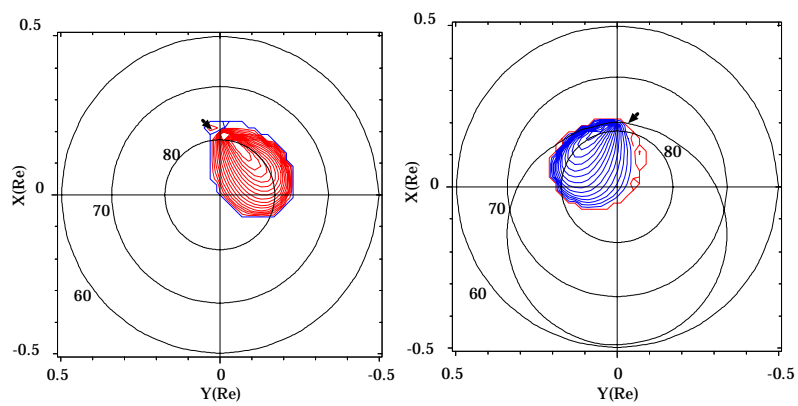

Fig. 9. Case 1. Left: The ionospheric electrostatic potential patterns in the northern polar cap are calculated at the time of case 1. The numbers denote the potential relative to the zero equipotential curves (which corresponds to the Sun-Earth line), with a contour separation of $16 \mathrm{kV}$. Only a single clockwise lobe cell shows up because a second minor counterclockwise lobe is very small for this event IMF direction. The arrow marks the ionospheric projection of the cusp. Right: The ionospheric electrostatic potential patterns in the southern polar cap are calculated for the case 1. Potential drop between two lines is $16 \mathrm{kV}$. The solid circle shows the auroral oval boundary calculated for $\mathrm{IMF}=0$. The equatorward boundary of the auroral oval corresponds to the inner edge of the tail current sheet. Only a single counterclockwise lobe cell shows up because a second minor clockwise lobe is very small for this event IMF direction. The arrow marks the ionospheric projection of the cusp.

The equipotentials located inside the regions bounded by the red and blue curves (Fig. 7) map to the convection lines of the polar caps. The open field lines that cross the magnetopause near the boundary marked by the red and blue curves in Fig. 7 map to the diffusion regions near the neutral points. The other open field lines, which intersect the magnetopause inside the open field line window, go far from the diffusion regions, and these field lines can be assumed to be equipotentials.

The small empty squares in Fig. 7 mark the positions of the magnetospheric cusps (magnetic field neutral points for $\mathrm{IMF}=0$ ). For $B_{y}>0$, the northern (southern) cusp moves duskward (dawnward) and into the magnetosphere for $B_{z}>0$.
The potential drop between the neutral points, $\delta \Phi_{\text {sep }}$, can be estimated as (see Fig. 7)

$\delta \Phi_{\mathrm{sep}}=\Phi_{O S}-\Phi_{O N}=2 R_{1} \kappa_{E} E_{\mathrm{IMFz}}$,

where $\Phi_{O S}$ and $\Phi_{O N}$ are electric potentials of the southern and northern neutral points, $R_{1}$ is the distance to the subsolar point of the magnetopause $\left(R_{1}=10 R_{E}\right.$, in the paraboloid model the distance along $Z$ from the equatorial plane to the cusp is of the order of $\left.R_{1}\right), E_{\mathrm{IMFz}}=B_{y} \cdot V$ is the northward component of the interplanetary electric field, and $\kappa_{E}$ describes the ratio of the ionospheric polar cap potential drop to the potential drop across the magnetosphere in the undisturbed solar wind (see Eq. 3). For both our events $\kappa_{E}=\kappa_{B} \kappa_{v}=0.05$, where $\kappa_{V}=0.5$ was assumed. In this case, Eq. (7) gives $\delta \Phi_{\mathrm{sep}}=0.1 R_{1} E_{\mathrm{IMFz}}$.

The points of intersections of the northern polar cap open field lines bundle with the equatorial plane are shown by crosses in Fig. 8 (the solar magnetospheric coordinate system is used). The empty (without crosses) tailward region in Fig. 8 is crossed by closed (interconnected both hemispheres) tail lobe field lines. One may consider a part of these very long field lines as belonging to the polar cap because they map to the dark polar ionosphere. We see that for this case, most of the northern open field lines cross the equatorial plane at the dawn side $($ at $y<0$ ). Only the lines nearest to the separator go to the dusk side near the subsolar equatorial plane. Almost all open field line bundles which cross- the equatorial plane are placed in the tail plasma sheet region. The nightside point of intersection of the separator with the equatorial plane is placed at $X=-90 R_{E}$ and $Y=-10$ $R_{E}$. The reconnection sites are projected to the boundary of the region covered by crosses. Open field lines appear at the sunward boundary of this region and then move in the antisunward direction and after intersecting with the tailward boundary transfer to the IMF lines.

Figure 9 shows the ionospheric electrostatic potential patterns in the northern and southern polar caps, respectively, calculated at the time of case 1 . For case 2 the same polar cap potential patterns are shown in Fig. 10. The numbers in Fig. 10 mark the distances along the electric field direction from the zero equipotential curve (which is chosen to coincide with the Sun-Earth line). These numbers are proportional to the corresponding potential values. The total potential drop in the northern polar cap can be calculated by Eq. (6). The total magnetospheric size in the dawn-dusk direction is about $74 R_{E}$ (see Fig. 8). In case 1 the pressure balance gives a subsolar distance $R_{1}=8.14 R_{E}$. The results demonstrate that the size of one of the open field line windows $L_{y}$ is $39 R_{E}$, or about half of the total magnetospheric size. In this case, Eq. (6) gives us $\Delta \Phi_{\mathrm{npc}}=156 \mathrm{kV}$ for the northern polar cap, in good agreement with the results of Blomberg et al. (2005), who studied two events illustrating the detailed relationships between plasma convection, field-aligned currents, and polar auroral emissions, as well as illustrating the influence of the IMF $B_{y}$ component 
on theta aurora development. Electric and magnetic field and precipitating particle data are provided by DMSP, while the POLAR UVI instrument provides measurements of auroral emissions. Ionospheric electrostatic potential patterns are calculated at different times during the evolution of the theta aurora using the KTH polar cap convection model. These model patterns have been compared to the convection predicted by mapping the magnetopause electric field to the ionosphere using the paraboloid model of the magnetosphere. The model results explain the correlation between TPA occurrence and the $B_{y}$ component of the IMF, because the potential drop on the separatrix is proportional to $B_{y}$. They support the observed TPA motion from dawn to dusk. In accordance with observations, the TPA region is collocated with sunward ionospheric convective flow.

For the field-aligned potential drop along the separator (between the neutral points), Eq. (7) gives $32 \mathrm{kV}$, a good agreement with the Siscoe et al. (2001) MHD simulations results. We can check our result by comparing with the polar cap potential drop which was determined by Korth et al. (2005). These authors presented a case study of a prolonged interval of strongly northward IMF on 16 July 2000. Based on magnetic field observations by the constellation of Iridium satellites, together with the drift meter and magnetometer observations from DMSP F13 and F15 satellites, and on the FUV images taken by the IMAGE spacecraft, Korth et al. (2005) estimated the electric potential drop as $45 \mathrm{kV}$. For the event interval (from 16:06 to 19:24 UT on 16 July 2000) the solar wind parameters were: $B_{t}=6.76 \mathrm{nT}, n=0.8 \mathrm{~cm}^{-3}$ and $V=740 \mathrm{~km} / \mathrm{s}$ (Korth et al., 2005), the total potential drop in the northern polar cap calculated by Eq. (6) is $53.49 \mathrm{kV}$, in good agreement with Korth et al. (2005). It is interesting to note that Korth et al. (2005) found that the potential drop associated with viscous interaction is less than $\sim 5 \mathrm{kV}$, or about 9 times smaller than the potential drop caused by high-latitude reconnection.

Figure 9 (right panel) shows the ionospheric electrostatic potential patterns in the southern polar cap calculated for case 1 . The equipotentials are shown in blue and they correspond to the southern polar cap. The total potential drop in the southern polar cap is $128 \mathrm{kV}$. In Fig. 9 (right panel) the black solid circle, which corresponds to the auroral oval equatorial boundary, is shown for comparison. The equatorward boundary of the auroral oval in our study corresponds to the inner edge of the tail current sheet.

We show in Figs. 9 and 10 only one convection cell. As a consequence of the method used here to calculate the convection lines, we can reproduce only the dominant lobe cell of a double lobe cell's system. A minor convection cell which exists on the left (right) side of the clockwise (counterclockwise) convection cell shown in the left panel of Fig. 9 and right panel of Fig. 10 (Fig. 9, right panel and 10, left panel) is not shown in these figures, since we would need a more complex calculation procedure for reproducing it. However, one should keep in the mind that really a two-cell
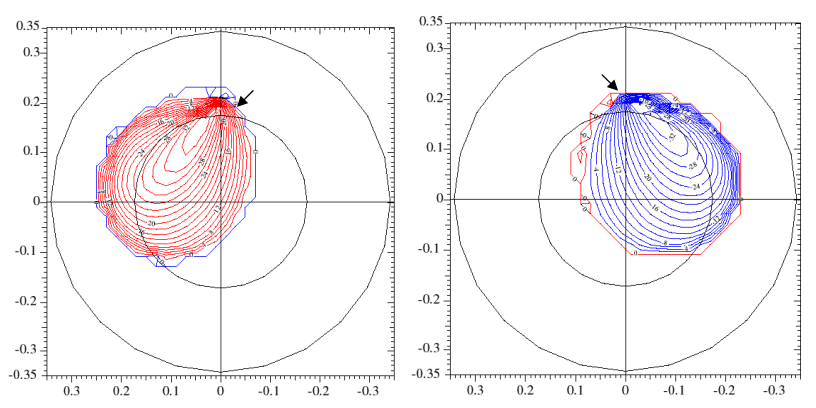

Fig. 10. Case 2. Left: Northern polar cap convection lines. Only a single clockwise lobe cell shows up because the second minor counterclockwise lobe is very small for this event IMF direction. The arrow marks the ionospheric projection of the cusp. Right: Southern polar cap convection lines are shown. Only a single clockwise lobe cell shows up because the second minor counterclockwise lobe is very small for this event IMF direction. The arrow marks the ionospheric projection of the cusp.

convection pattern with one cell much larger than the other exists in the open field line region in the polar cap for northward IMF with $B_{x} \neq 0$.

As one can see from Fig. 7 (bottom panel), for case 2 the transverse magnetospheric size $L_{y}$ along the solar wind electric field direction is about $70 R_{E}$, in good agreement with the estimation $8 R_{1}=63.2 R_{E}$ by Alexeev et al. (2000). Similar to case 1 , the calculation results demonstrate that the size of one of the open field line windows is $40 R_{E}$ or about half of the total magnetospheric size. Finally, we have $\Delta \Phi_{\mathrm{npc}}=157 \mathrm{kV}$ for the northern polar cap and $\Delta \Phi_{\mathrm{spc}}=150 \mathrm{kV}$ for the southern polar cap. For the fieldaligned potential drop along the separator (between the neutral points), Eq. (7) gives $32 \mathrm{kV}$, which is the same as for case 1 . The solar wind electric fields and subsolar distances are almost equal for both these cases (ratio $E_{s w 1} / E_{s w 2}$ is equal to 1.02 , and $R_{11} / R_{12}$ is equal to 1.03 ).

\section{Summary}

We studied here the modeled structure of the open field line bundles for northward IMF and forced solar wind conditions, which were observed during an event lasting several hours on 8 November 1998. Two cases, which were used for our analysis, correspond to opposite directions of the IMF $B_{y}$ component.

Our results support the previous finding about a displacement of the ionospheric reconnection site projection (cusp) in the $B_{y}\left(-B_{y}\right)$ direction for the northern (southern) polar cap (see, for example, Belenkaya, 1998b). The ionospheric open field line region shifts in the opposite direction. For the Northern Hemisphere our model predicts a convection change from a single clockwise lobe cell to a single counterclockwise lobe cell via an intermediate double lobe cell 
system when the IMF changes from positive (case 1) to negative IMF $B_{y}$ (case 2) as during this event (in the 12:4015:30 UT period on this day).

We obtained estimates of the polar cap potential drops in both hemispheres. Model calculations showed that the northern and southern potential drops can be different if $B_{x}$ is comparable with $B_{y}$. If $B_{x}<0$, the potential drop is higher in the preferred northern polar cap, which is directly connected with the Sun by open field lines.

For northward IMF there are two connection points with the magnetosphere. In one cusp region, the IMF reconnects with closed field lines and forms open field lines, which are partially inside the magnetosphere and partially in interplanetary space. In the other cusp region, open field lines of both polar caps form interplanetary and closed field lines.

For the southward IMF case, the northern and southern open field line windows at the magnetopause touch each other at the reconnection line (separator). The separator is placed in the magnetopause, inside the current sheet. Contrary to it, for the northward IMF case, the northern and southern open field line windows at the magnetopause never touch each other. Between these windows the magnetopause is intersected by the IMF, which goes in and out of the magnetosphere. Inside the magnetosphere an IMF layer is formed.

Such a structure of the open field line bundles and the inner reconnection sites may explain why for the northward IMF the polar cap potential drop is about four times smaller than that for the southward IMF case, for the same value of the upstream solar wind electric field. We have demonstrated two reasons for the attenuation of the electric field inside the magnetosphere for northward IMF. First, the diameter of the open field line bundle is about half of the total size of the magnetosphere in the direction perpendicular to the electric field. The second reason for the reduction in the solar wind electric field inside the magnetosphere is the internal position of the reconnection site. It is located at some distance inside the magnetosphere away from the magnetopause, and the flow velocity in this region is about half of the undisturbed solar wind velocity.

Acknowledgements. We thank the ACE MAG and SWEPAM instrument teams and the ACE Science Center for providing the ACE data. The work at the Moscow State University was partially supported by the RFBR Grants 05-05-64435, 06-05-64508, and 07-0500529. Work at the Royal Institute of Technology was partially supported by the Swedish National Space Board and the Alfvén Laboratory Centre for Space and Fusion Plasma Physics. Work at the University of Texas at Dallas was supported by NSF grant ATM0536868 and NASA grant NNG04GG84G.

Topical Editor I. A. Daglis thanks J. Dorelli and and another anonymous referee for their help in evaluating this paper.

\section{References}

Alexeev, I. I.: The penetration of interplanetary magnetic and electric fields into the magnetosphere, J. Geomagn. Geoelectr., 38, 1199-1221, 1986.

Alexeev, I. I.: What define the polar cap and auroral oval diameter?, in: The Inner Magnetosphere: Physics and Modeling, edited by: Pulkkinen, T. I., Tsyganenko, N. A., and Friedel, R. H. W.: Geophysical Monograph Series, Volume 155, 313 pp., 257-262, ISBN 0-87590-420-3, AGU Code GM1554203, 2005.

Alekseyev, I. I. and Belenkaya, E. S.: Electric field in an open model of the magnetosphere, Geomagn. Aeron., Engl. Transl., 23, 57$61,1983$.

Alexeev, I. I. and Belenkaya, E. S., Magnetospheric plasma convection on the open field lines, Geomagn. Aeron., 25, 450-457, 1985 (in Russian).

Alexeev, I.I. and Belenkaya, E. S.: Magnetospheric convection structure for the southward and northward IMF, Geomagn. Aeron., 29, 725-729, 1989 (in Russian).

Alexeev, I. I. and Kalegaev, V. V.: Magnetic field and the plasma flow structure near the magnetopause, J. Geophys. Res., 100(10), 19267-19276, 1995.

Alexeev, I. I., Belenkaya, E. S., and Clauer, C. R.: A model of region 1 field-aligned currents dependent on ionospheric conductivity and solar wind parameters, J. Geophys. Res., 105, 21 119$21127,2000$.

Alexeev, I. I., Sibeck, D. G., and Yu, S.: Bobrovnikov, Concerning the location of magnetopause merging as a function of the magnetopause current strength, J. Geophys. Res., 103(A4), 66756684, 1998.

Alexeev, I. I., Belenkaya, E. S., Bobrovnikov, S. Yu., and Kalegaev, V. V.: Modelling of the electromagnetic field in the interplanetary space and in the Earth's magnetosphere, Space Sci. Rev., 107, 726, 2003.

Belenkaya E. S.: Comment on "Observations of reconnection of interplanetary and lobe magnetic field lines at the high-latitude magnetopause" by Gosling, J. T., Thomsen, M. F., Bame, S. J., Elphic, R. C., and Russell, C. T., J. Geophys. Res., 98(A4), 5941-5944, 1993.

Belenkaya, E. S.: Reconnection modes for near-radial IMF, J. Geophys. Res., 103(A011), 26487-26494, 1998a.

Belenkaya, E. S.: High-latitude ionospheric convection patterns dependent on the variable IMF orientation, J. Atmos. Solar-Terr. Phys., 60/13, 1343-1354, 1998b.

Belenkaya, E. S., Alexeev, I. I., and Clauer, C. R.: Field-aligned current distribution in the transition current system, J. Geophys. Res., 109, A11207, doi:10.1029/2004JA010484, 2004.

Belenkaya E. S.: The Jovian magnetospheric magnetic and electric fields: effects of the interplanetary magnetic field, Planet. Space Sci., 52/5-6, 499-511, 2004.

Belenkaya, E. S., Cowley, S. W. H., and Alexeev, I. I.: Saturn's aurora in the January 2004 events, Ann. Geophys., 24, 16491663, 2006a.

Belenkaya, E. S., Bespalov, P. A., Davydenko, S. S., and Kalegaev, V. V.: Magnetic field influence on aurorae and the Jovian plasma disk radial structure, Ann. Geophys., 24, 973-988, 2006b.

Blomberg, L. G., Cumnock, J. A., Alexeev, I. I., Belenkaya, E. S., Bobrovnikov, S. Yu., and Kalegaev, V. V.: Transpolar aurora: Time evolution, associated convection patterns, and a possible cause, Ann. Geophys., 23, 1917-1930, 2005, 
http://www.ann-geophys.net/23/1917/2005/.

Bogdanova, Y. V., Marchaudon, A., Owen, C. J., Dunlop, M. W., Frey, H. U., Wild, J. A., Fazakerley, A. N., Klecker, B., Davies, J. A., and Milan, S. E.: On the formation of the high-altitude stagnant cusp: Cluster observations, Geophys. Res. Lett., 32, L12101, doi:10.1029/2005GL022813, 2005.

Carlson, H. C. and Cowley, S. W. H.: Accelerated polar rain electrons as the source of Sun-aligned arcs in the polar cap during northward interplanetary magnetic field conditions, J. Geophys. Res., 110, A05302, doi:10.1029/2004JA010669, 2005.

Cowley, S. W. H.: A qualitative study of the reconnection between the Earth's magnetic field and an interplanetary field of arbitrary orientation, Radio Sci., 8(11), 903-913, 1973.

Cowley, S. W. H.: Interpretation of observed relations between solar-wind characteristics and effects at ionospheric altitudes, in: High Latitude Space Plasma Physics, edited by: Hultqvist, B. and Hagfors, T., 225-249, Plenum, New York, 1983.

Crooker, N. U.: Morphology of magnetic merging at the magnetopause, J. Atmos. Terr. Phys., 52, 1123-1134, 1990.

Crooker, N. U.: Reverse convection, J. Geophys. Res., 97, $19363-$ 19372, 1992.

Crooker, N. U., Siscoe, G. L., and Toffoletto, F. R.: A tangent subsolar merging line, J. Geophys. Res., 95, 3787-3793, 1990.

Crooker, N. U., Lyon, J. G., and Fedder, J. A.: MHD model merging with IMF BY: Lobe cells, sunward polar cap convection, and overdraped lobes, J. Geophys. Res., 103, 9143-9151, 1998.

Cumnock, J. A., Sharber, J. R., Heelis, R. A., Hairston, M. R., and Craven, J. D.: Evolution of the global aurora during positive IMF Bz and varying IMF By conditions, J. Geophys. Res., 102, 17 489-17 498, 1997.

Cumnock, J. A. and Blomberg, L. G.: Transpolar arc evolution and associated potential patterns, Ann. Geophys., 22, 1213-1231, 2004,

http://www.ann-geophys.net/22/1213/2004/.

Cumnock, J. A.: High-latitude aurora during steady northward interplanetary magnetic field and changing IMF By, J. Geophys. Res., 110(A2), A02304, doi: 10.1029/2004JA010867, 2005.

Cumnock, J. A., Blomberg, L. G., Alexeev, I. I., Belenkaya, E. S., Bobrovnikov, S. Yu., and Kalegaev, V. V.: Simultaneous polar aurorae and modelled convection patterns in both hemispheres, Adv. Space Res., 38, 1685-1693, 2006.

Dorelli, J. C., Hesse, M., Kuznetsova, M. M., Rastaetter, L., and Raeder, J.: A new look at driven magnetic reconnection at the terrestrial subsolar magnetopause, J. Geophys. Res., 109, A12216, doi:10.1029/2004JA010458, 2004.

Dungey, J. W.: The structure of the exosphere or adventures in velocity space, in: Geophysics, The Earth's Environment, edited by: DeWitt, C., Hieblot, J., and LeBeau, L., 503-550, Gordon and Breach, New York. 1963.

Frank, L. A., Craven, J. D., Burch, J. L., and Winningham, J. D.: Polar views of the Earth s aurora with Dynamics Explorer, Geophys. Res. Lett., 9, 1001-1004, 1982.
Frank, L. A., Craven, J. D., Gurnett, D. A., Shawhan, S. D., Weimer, D. R., Burch, J. L., Winningham, J. D., Chappell, C. R., Waite, J. H., Heelis, R. A., Maynard, N. C., Sugiura, M., Peterson, W. K., and Shelley, E. C.: The theta aurora, J. Geophys. Res., 91, 3177-3224, 1986.

Korth, H., Anderson, B. J., Frey, H. U., and Waters, C. L.: Highlatitude electromagnetic and particle energy flux during an event with sustained strongly northward IMF, Ann. Geophys., 23, 1295-1310, 2005, http://www.ann-geophys.net/23/1295/2005/.

Kullen, A., Brittnacher, M., Cumnock, J. A., and Blomberg, L. G.: Solar wind dependence of the occurrence and motion of polar auroral arcs: A statistical study, J. Geophys. Res., 107, 1362, doi:10.1029/2002JA009245, 2002.

Kullen, A. and Janhunen, P.: Relation of polar auroral arcs to magnetotail twisting and IMF rotation: A systematic MHD simulation study, Ann. Geophys., 22, 951-970, 2004, http://www.ann-geophys.net/22/951/2004/.

Lyons, L.: Generation of large-scale regions of auroral currents, electric potentials, and precipitation by the divergence of the convection electric field, J. Geophys. Res., 85, 17-25, 1980.

Maynard, N. C., Burke, W. J., Moen, J., et al.: Responses of the open - closed field line boundary in the evening sector to IMF changes: A source mechanism for Sun-aligned arcs, J. Geophys. Res., 108(A1), 1006, doi:10.1029/2001JA000174, 2003.

Peterson, W. K. and Shelley, E. G.: Origin of the plasma in a cross polar cap auroral feature (theta aurora), J. Geophys. Res., 89, 6729-6735, 1984.

Reiff, P. H. and Burch, J. L.: IMF By-dependent on dayside plasma flow and Birkeland currents in the dayside magnetosphere: 2. A global model for northward and southward IMF, J. Geophys. Res., 90, 1595-1611, 1985.

Siscoe, G. L.: The magnetospheric boundary, in Physics of Space Plasmas (1987), edited by: Chang, T., Crew, G. B., and Jasperse, J. R., 3-78, Sci. Publ., Inc., Cambridge, Mass., 1988.

Siscoe, G. L., Erickson, G. M., Sonnerup, B. U. Ö., Maynard, N. C., Siebert, K. D., Weimer, D. R., and White, W. W.: Global role of Ek in magnetopause reconnection: An explicit demonstration, J. Geophys. Res., 106, 13 015-13 022, 2001.

Toffoletto, F. R. and Hill, T. W.: Mapping of the solar wind electric field to the Earth's polar caps, J. Geophys. Res., 94(A1), 329347, 1989.

Vennerstrom, S., Moretto, T., Rastatter, L., and Raeder, J.: Field-aligned currents during northward interplanetary magnetic field: Morphology and causes, J. Geophys. Res., 110, A06205, doi:10.1029/2004JA010802, 2005.

Watanabe, M., Kabin, K., Sofko, G. J., Rankin, R., Gombosi, T. I., Ridley, A. J., and Clauer, C. R.: Internal reconnection for northward interplanetary magnetic field, J. Geophys. Res., 110, A06210, doi:10.1029/2004JA010832, 2005.

Yamamoto, T. and Ozaki, M.: A numerical model of the dayside aurora, J. Geophys. Res., 110, A05215, doi:10.1029/2004JA010786, 2005. 